\title{
Effectiveness of medicines review with web- based pharmaceutical treatment algorithms in reducing potentially inappropriate prescribing in older people in primary care: a cluster randomized trial (OPTI-SCRIPT study protocol)
}

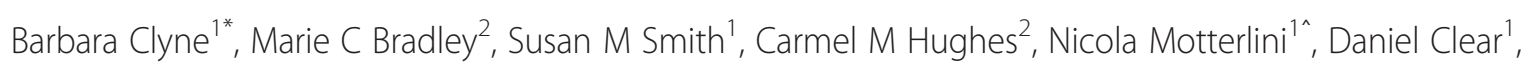
Ronan McDonnell ${ }^{1}$, David Williams ${ }^{3}$, Tom Fahey ${ }^{1}$ and on behalf of the OPTI-SCRIPT study team

\begin{abstract}
Background: Potentially inappropriate prescribing in older people is common in primary care and can result in increased morbidity, adverse drug events, hospitalizations and mortality. In Ireland, 36\% of those aged 70 years or over received at least one potentially inappropriate medication, with an associated expenditure of over $€ 45$ million. The main objective of this study is to determine the effectiveness and acceptability of a complex, multifaceted intervention in reducing the level of potentially inappropriate prescribing in primary care.

Methods/design: This study is a pragmatic cluster randomized controlled trial, conducted in primary care (OPTISCRIPT trial), involving 22 practices (clusters) and 220 patients. Practices will be allocated to intervention or control arms using minimization, with intervention participants receiving a complex multifaceted intervention incorporating academic detailing, medicines review with web-based pharmaceutical treatment algorithms that provide recommended alternative treatment options, and tailored patient information leaflets. Control practices will deliver usual care and receive simple patient-level feedback on potentially inappropriate prescribing. Routinely collected national prescribing data will also be analyzed for nonparticipating practices, acting as a contemporary national control. The primary outcomes are the proportion of participant patients with potentially inappropriate prescribing and the mean number of potentially inappropriate prescriptions per patient. In addition, economic and qualitative evaluations will be conducted.
\end{abstract}

Discussion: This study will establish the effectiveness of a multifaceted intervention in reducing potentially inappropriate prescribing in older people in Irish primary care that is generalizable to countries with similar prescribing challenges.

Trial registration: Current controlled trials ISRCTN41694007

Keywords: Multifaceted intervention, Potentially inappropriate prescribing, Primary care, Randomized controlled trial

\footnotetext{
* Correspondence: barbaraclyne@rcsi.ie

Deceased

${ }^{1}$ Health Research Board (HRB) Centre for Primary Care Research, Royal College of Surgeons in Ireland (RCSI), Beaux Lane House, Lower Mercer Street, Dublin, Ireland

Full list of author information is available at the end of the article
} 


\section{Background}

\section{Prescribing in older people}

Older people are among the biggest consumers of healthcare services, particularly drug therapy [1]. They tend to have multiple conditions, requiring multiple drug treatments [2]. They also experience age-related changes in physiology and body composition that influence the body's ability to process medications efficiently, in terms of both pharmacokinetics (the body's ability to absorb, distribute, metabolize and excrete a drug) and pharmacodynamics (the drug's physiological effects) $[3,4]$. Thus, prescribing for older people is a complex and challenging task, with the potential for adverse outcomes including drug-drug interactions, adverse drug reactions and potentially inappropriate prescribing (PIP) [5].

The term 'potentially inappropriate prescribing' covers a number of suboptimal prescribing practices, particularly the use of medicines that introduce a greater risk of adverse drug-related events where a safer and equally effective alternative is available to treat the same condition [6]. Inappropriate prescribing in older people can result in increased morbidity, adverse drug events, hospitalizations and mortality [7-9]. Potentially inappropriate prescribing may be measured with explicit (criterion-based) or implicit (judgment-based) tools [9]. A recently developed explicit process measure, the Screening Tool of Older People's Prescriptions (STOPP), has been published for use in European settings [10].

When these criteria were applied to an Irish pharmacy claims database (containing the prescription records of $97 \%$ of those aged $\geq 70$ nationally), it was found that $36 \%$ of those aged $\geq 70$ years received at least one potentially inappropriate medication. Total PIP expenditure was estimated at over $€ 45$ million (or $9 \%$ of expenditure on pharmaceuticals in that age group) [11]. The clinical and economic burden of PIP is an important public health concern and it is important to minimize PIP where possible, to increase patient safety and encourage costeffective prescribing.

\section{Interventions to change prescribing}

Changing professional practice is a complex and difficult task. While the usefulness of behaviour change theory in intervention and implementation research has been questioned, $[12,13]$ there is general consensus that interventions are more likely to have an impact when they target all stages of behaviour change [14]. Studies to date have yielded mixed results in terms of which intervention types are most effective in improving prescribing and there is no one interventional strategy that has proved to be most effective [15]. Strategies shown to be effective for improving prescribing outcomes include educational outreach visits (academic detailing) [16] and interventions involving a pharmacist [17]. Pharmacist services, such as conducting medicines reviews or providing advice to general practitioners (GPs) may lead to improvements in prescribing outcomes, including more appropriate prescribing in older people [9,17-21]. Patient-mediated interventions do not consistently show effects in improving prescribing [22]; however, evidence suggests that providing patients with information is important. Patient information leaflets may be helpful in improving patient outcomes, and older people appreciate receiving brief, clearly written information leaflets $[23,24]$. A number of commentators have argued that a multifaceted intervention, an approach that combines a number of techniques within a single intervention [20], may be more likely to work than any one single intervention $[9,25,26]$.

\section{Study aim and objectives}

The aim of this study is to determine the effectiveness and acceptability of a complex, multifaceted intervention in reducing the level of PIP in primary care. The intervention combines academic detailing, medicines review with web-based pharmaceutical treatment algorithms that provide recommended alternative treatment options, and tailored patient information leaflets. The intervention development was informed by the Medical Research Council (MRC) guidelines for the development and evaluation of randomized controlled trials (RCTs) $[27,28]$. It was piloted with a group of five GPs and found to be feasible and acceptable within this group.

Secondary objectives are to evaluate the effect of the intervention on patient outcomes in terms of the number of GP visits, the number of hospitalizations, patient well-being, beliefs about medicines and health status. The views and experiences of participants concerning the possible reasons for the intervention to be effective or ineffective will be explored using qualitative methodology. In addition, an economic evaluation will be carried out.

\section{Methods/design \\ Trial design}

The OPTI-SCRIPT study is a pragmatic two-arm cluster $\mathrm{RCT}$, incorporating qualitative analysis. Qualitative approaches can contribute in several ways to the development and evaluation of health interventions; they have been used during intervention development and piloting and will be used to undertake a process evaluation of the trial [29]. The study design was developed in line with the Consolidated Standards of Reporting Trials (CONSORT) statement extension to cluster RCTs [30]. A cluster design was chosen to avoid the possibility of contamination across arms. A GP who is treating both intervention and control patients might find it difficult 


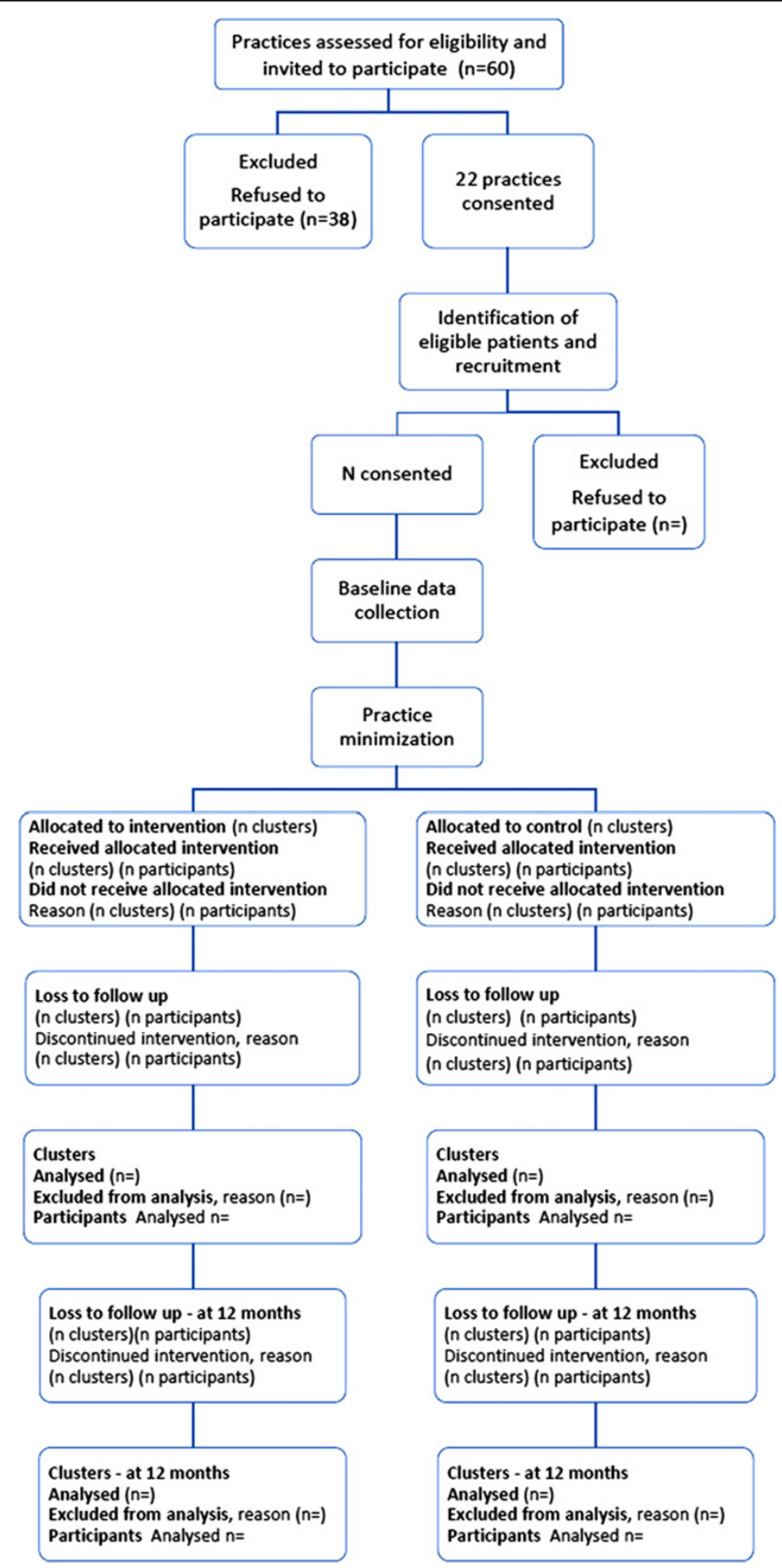

Figure 1 Flow of practices and patients through RCT. 
to behave differently towards each group [31]. The trial will be conducted in primary care with GP practices as the unit of randomization. A practice can be a singlehanded GP or can comprise two or more GPs (group practices); however, not all GPs in group practices may wish to participate. Participation may be decided by one GP on behalf of the group practice, in which case that GP will be the main point of contact for the study. We will keep a record of participating GPs. Participation in the intervention arm will be defined as attendance at the academic detailing visit and undertaking medicines reviews, while participation in the control group as providing patient prescription data. Practices that do not meet these criteria will be considered lost to follow-up (see Figure 1). The intervention will be delivered at the GP level while the unit of analysis will be at the individual patient level, in terms of medicines prescribed, adjusting for clustering.

\section{Study population}

Practices are eligible to participate if they have approximately 80 or more older patients (aged $\geq 70$ years) on their patient panel (based on the need to recruit $10 \mathrm{pa}-$ tients per practice, and allowing for refusals) and they are based in the greater Dublin area (to facilitate the academic detailing process).

Practices are excluded where they have been involved in the development and piloting of the intervention or other concurrent medication quality related studies.

Patients will be considered eligible if they are aged 70 years or over and they are being prescribed one or more selected potentially inappropriate prescriptions on a repeat basis (only patients with an existing PIP will be included, as the intervention is specifically targeting the management of PIP).

Patients will be excluded if they have significant mental or physical illness that is likely to impair their ability to participate in the study, or they are unable to attend the GP surgery for consultation (for example, they are nursing home residents) or they are participating in another medication quality related study.

\section{Recruitment and allocation}

Potentially eligible practices (based in the greater Dublin area) will be identified from the Health Research Board (HRB) Primary Care Research Centre research network. All eligible practices will be invited to participate by an email (or letter where email address is unavailable),

Table 1 Intervention components

\begin{tabular}{|c|c|}
\hline Intervention component & Description \\
\hline \multirow[t]{5}{*}{ Academic detailing } & $\begin{array}{l}\text { A research pharmacist will visit the intervention practices. During the academic detailing, the } \\
\text { pharmacist will: }\end{array}$ \\
\hline & $\begin{array}{l}\text { - Discuss the concept of PIP with the GPs, focusing on the prevalence and consequences of PIP in } \\
\text { primary care }\end{array}$ \\
\hline & - Discuss the pharmaceutical treatment algorithm \\
\hline & - Discuss the medicines review process \\
\hline & $\begin{array}{l}\text { - Demonstrate the web-based platform for accessing the pharmaceutical treatment algorithm for } \\
\text { use in a medicines review with each participant patient }\end{array}$ \\
\hline \multirow{10}{*}{$\begin{array}{l}\text { Medicines review with web-based } \\
\text { pharmaceutical treatment algorithms }\end{array}$} & GPs will be asked to: \\
\hline & - Schedule a medicines review for the patient's next appointment \\
\hline & - Log on to the designated website using individualized user-names and passwords \\
\hline & $\begin{array}{l}\text { - Access the individualized web-based pharmaceutical treatment algorithms for each patient } \\
\text { during the review }\end{array}$ \\
\hline & $\begin{array}{l}\text { - Conduct a medicines review following the page-by-page web-based pharmaceutical treatment } \\
\text { algorithms. Each pharmaceutical treatment algorithm has the following structure: }\end{array}$ \\
\hline & Section A: The individual PIP with reason for concern \\
\hline & Section B: Alternative pharmacological and nonpharmacological treatment options \\
\hline & Section C: Background information (where relevant) \\
\hline & - Complete the process by submitting the review outcome form for each PIP per patient \\
\hline & $\begin{array}{l}\text { Each GP will also be provided with a full, paper-based compendium of pharmaceutical treatment } \\
\text { algorithms for reference }\end{array}$ \\
\hline Patient information leaflets & $\begin{array}{l}\text { For every alternative therapy option, a brief patient information leaflet has been written. These } \\
\text { leaflets describe the PIP and the reasons why it may be inappropriate. They also outline the } \\
\text { alternative therapies the GP may offer instead }\end{array}$ \\
\hline
\end{tabular}


Table 2 Selected prescribing criteria and indicators

\begin{tabular}{l} 
Criterion \\
\hline PPI for peptic ulcer disease at full therapeutic dosage for $>8$ weeks \\
NSAID (>3 months) for relief of mild joint pain in osteoarthritis \\
Long-term (>1 month), long-acting benzodiazepines, for example, \\
chlordiazepoxide, flurazepam, nitrazepam and clorazepate, and \\
benzodiazepines with long-acting metabolites, for example, \\
diazepam \\
Any regular duplicate drug class prescription, for example, two \\
concurrent opiates, NSAIDs, SSRIs, loop diuretics, ACE inhibitors. \\
Excludes duplicate prescribing of drugs that may be required on a \\
p.r.n. basis, for example, inhaled $\beta 2$ agonists (long and short acting) \\
for asthma or COPD, and opiates for management of breakthrough \\
pain \\
TCAs with an opiate or calcium channel blocker
\end{tabular}

Aspirin at dosage $>150 \mathrm{mg} /$ day

Theophylline as monotherapy for COPD or asthma

Use of aspirin and warfarin in combination without histamine $\mathrm{H}_{2}$ receptor antagonist (except cimetidine because of interaction with warfarin) or PPI

Doses of short-acting benzodiazepines, doses greater than: lorazepam $\left(\right.$ Ativan $\left.^{\circledR}\right)$, 3 mg; oxazepam $\left(\right.$ Serax $\left.^{(}\right)$), 60 mg; alprazolam $\left(\right.$ Xanax $\left.^{\circledR}\right), 2$ mg; temazepam (Restoril $\left.{ }^{\circledR}\right), 15$ mg; and triazolam $\left(\right.$ Halcion $\left.^{\circledR}\right), 0.25 \mathrm{mg}$

Prolonged use ( $>1$ week) of first-generation antihistamines, that is, diphenhydramine, chlorpheniramine, cyclizine, promethazine

Warfarin and NSAID together

Calcium channel blockers with chronic constipation

NSAID with history of peptic ulcer disease or Gl bleeding, unless with concurrent histamine $\mathrm{H}_{2}$ receptor antagonist, PPI or misoprostol

Bladder antimuscarinic drugs with dementia

TCAs with constipation

Digoxin at a long-term dosage $>125 \mu \mathrm{g}$ /day (with impaired renal function)

\section{Concern}

Prevalence

in Ireland*

Earlier discontinuation or dose reduction for maintenance or prophylactic treatment of peptic ulcer disease, oesophagitis or GORD is indicated

$16.69 \%[11]$

$7.68 \%[49]$

$4.06 \%[10]$

Simple analgesics are preferable and usually as effective for pain relief

$8.76 \%[11]$

$1.05 \%$ [49]

$1.26 \%[10]$

Risk of prolonged sedation, confusion, impaired balance, falls

$5.22 \%[11]$

$5.19 \%[49]$

$3.00 \%$ [49]

$6.01 \%[10]$

$9.09 \%[10]$

Optimization of monotherapy within a single drug class should

$4.78 \%[11]$

be observed prior to considering a new class of drug

$2.18 \%[49]$

$6.01 \%[10]$

Risk of severe constipation

$2.05 \%[11]$

$0.37 \%[49]$

Increased bleeding risk, no evidence for increased efficacy

$1.69 \%[11]$

$0.3 \%[49]$

$0.14 \%[10]$

Risk of adverse effects due to narrow therapeutic index

$1.18 \%[11]$

$0.56 \%[10]$

High risk of $\mathrm{Gl}$ bleeding

$1.09 \%[11]$

$0.3 \%[49]$

Total daily doses should rarely exceed the suggested maximums

$0.98 \%$ [49]

$1.54 \%[10]$

$0.96 \%[11]$

$0.15 \%$ [49]

$0.75 \%[11]$

$1.68 \%[10]$

May exacerbate constipation

$0.68 \%$ [49]

$0.28 \%$ [10]

Risk of peptic ulcer relapse

$0.67 \%$ [49]

$0.42 \%[10]$

Risk of increased confusion, agitation

$0.46 \%[11]$

$0.84 \%$ [10]

May worsen constipation

$0.45 \%$ [49]

$0.14 \%[10]$

$0.36 \%$ [11]

$0.15 \%$ [49]

$0.55 \%[10]$

May exacerbate gout 
Table 2 Selected prescribing criteria and indicators (Continued)

\begin{tabular}{|c|c|c|}
\hline & & $0.45 \%[49]$ \\
\hline & & $0.14 \%[10]$ \\
\hline \multirow[t]{2}{*}{ Glibenclamide (with type 2 diabetes mellitus) } & \multirow[t]{2}{*}{ Risk of prolonged hypoglycaemia } & $0.29 \%[11]$ \\
\hline & & $0.22 \%[49]$ \\
\hline \multirow{2}{*}{$\begin{array}{l}\text { Aspirin with a past history of peptic ulcer disease, without histamine } \\
\mathrm{H} 2 \text { receptor antagonist or PPI }\end{array}$} & \multirow{2}{*}{ Risk of bleeding } & $0.22 \%[49]$ \\
\hline & & $0.28 \%[10]$ \\
\hline Prochlorperazine $\left(\right.$ Stemetil $\left.{ }^{\circledR}\right)$ or metoclopramide with parkinsonism & Risk of exacerbating parkinsonism & $0.21 \%[11]$ \\
\hline \multirow[t]{2}{*}{ TCAs with dementia } & \multirow[t]{2}{*}{ Risk of worsening cognitive impairment } & $0.18 \%[11]$ \\
\hline & & $0.28 \%[10]$ \\
\hline \multirow[t]{2}{*}{ TCAs with glaucoma } & \multirow[t]{2}{*}{ Likely to exacerbate glaucoma } & $0.14 \%[11]$ \\
\hline & & $0.07 \%[49]$ \\
\hline TCAs with cardiac conductive abnormalities & Pro-arrhythmic effects & $0.14 \%[10]$ \\
\hline $\begin{array}{l}\text { Long-term corticosteroids (>3 months) as monotherapy for } \\
\text { rheumatoid arthritis or osteoarthritis }\end{array}$ & Risk of major systemic corticosteroid side-effects & $0.14 \%[10]$ \\
\hline Bladder antimuscarinic drugs with chronic prostatism & Risk of urinary retention & $0.14 \%[10]$ \\
\hline \multirow[t]{2}{*}{ NSAID with heart failure } & \multirow[t]{2}{*}{ Risk of exacerbation of heart failure } & $0.07 \%[49]$ \\
\hline & & $0.14 \%[10]$ \\
\hline \multirow[t]{2}{*}{ TCAs with prostatism or prior history of urinary retention } & \multirow[t]{2}{*}{ Risk of urinary retention } & $0.07 \%[49]$ \\
\hline & & $0.14 \%[10]$ \\
\hline \multirow{2}{*}{$\begin{array}{l}\text { Systemic corticosteroids instead of inhaled corticosteroids for } \\
\text { maintenance therapy in COPD or asthma }\end{array}$} & \multirow{2}{*}{$\begin{array}{l}\text { Unnecessary exposure to long-term side-effects of systemic } \\
\text { steroids }\end{array}$} & $0.07 \%[49]$ \\
\hline & & $0.56 \%[10]$ \\
\hline Bladder antimuscarinic drugs with chronic glaucoma & Risk of acute exacerbation of glaucoma & $<0.01 \%[11]$ \\
\hline NSAID with SSRI & Increased risk of Gl bleeding & N/A \\
\hline Bladder antimuscarinic drugs with chronic constipation & Risk of exacerbation of constipation & $\mathrm{N} / \mathrm{A}$ \\
\hline $\begin{array}{l}\text { Prednisolone (or equivalent) }>3 \text { months or longer without } \\
\text { bisphosphonate }\end{array}$ & Increased risk of fracture & $\mathrm{N} / \mathrm{A}$ \\
\hline NSAID with ACE-inhibitor & $\begin{array}{l}\text { Risk of kidney failure, particularly with the presence of general } \\
\text { arteriosclerosis, dehydration or concurrent use of diuretics }\end{array}$ & N/A \\
\hline NSAID with diuretic & $\begin{array}{l}\text { May reduce the effect of diuretics and worsen existing heart } \\
\text { failure }\end{array}$ & N/A \\
\hline
\end{tabular}

ACEl, angiotensin-converting-enzyme inhibitor; COPD, chronic obstructive pulmonary disease; Gl, gastro-intestinal; GORD, gastro-oesophageal reflux disease; N/A, not available; NSAID, nonsteroidal anti-inflammatory drug; PPI, proton pump inhibitor; p.r.n., pro re nata, as needed; SSRI, selective serotonin reuptake inhibitor; TCA, tricyclic anti-depressant. *Prevalence: the proportion of the study population with one or more potentially inappropriate medications.

which will include a study information leaflet outlining steps of the intervention and availability of continuing medical education (CME) points for participation. When a practice agrees to participate, a member of staff (for example, a GP, practice manager or nurse) will be selected by the practice. The research team will instruct the designated person on how to identify a random sample of 50 patients aged 70 years and over from the patients of participating GPs within the practice. They will pseudo-anonymize the records by assigning the patients a study ID and send a copy of the pseudo-anonymized prescription records to the research team, where a research pharmacist will generate a list of potentially eligible patients, that is, patients with PIP. A maximum of ten patients per practice is required; if more than ten patients are identified, ten patients will be selected at random from the list of eligible patients. Eligible patients will be sent a letter of invitation, a patient information leaflet and a consent form, asking them to participate and answer a questionnaire. Prior to practice allocation, baseline patient data (including prescription data, process-of-care measures and patient-reported outcomes - see sections on outcomes and data collection) will be collected. Practices will then be assigned to intervention or control using minimization. This approach offers the advantage of ensuring balance between the groups [32] in terms of prognostic factors: in this case, practice size (number of whole-time-equivalent GPs) and practice location (urban or rural, where an urban area is defined as a relatively small centre of population, with 5000 or more residents [33]). A chart of the flow of participants through the study is presented in Figure 1. Because of 
Table 3 Secondary outcome measures

\begin{tabular}{ll}
\hline Secondary outcome & Measure \\
\hline $\begin{array}{l}\text { Drug-specific } \\
\text { outcomes }\end{array}$ & The absolute number of PIPs per patient of the top five occurring PIP drugs: [1 1] \\
& Proton pump inhibitor (PPI) for peptic ulcer disease at full therapeutic dosage for >8 weeks \\
& Long-term (>3 months) use of NSAIDs for relief of mild joint pain in osteoarthritis \\
& $\begin{array}{l}\text { Long-term (>1 month) use of long-acting benzodiazepines, for example, chlordiazepoxide, flurazepam, nitrazepam, } \\
\text { chlorazepate and benzodiazepines with long-acting metabolites for example, diazepam }\end{array}$ \\
& Any regular duplicate drug class prescription \\
& TCAs with an opiate or calcium channel blocker \\
& Mean number of PIPs per patient of the top five PIP drugs (as above) \\
Patient-reported & Health status (EQ-5D) \\
outcomes & Patients' Beliefs about Medicine Questionnaire (BMQ) \\
Process-of-care & Well-being Questionnaire (WBQ-12) \\
measures & Number of GP visits (6 months prior to enrolment and at 4 and 12 month follow-up) \\
Process evaluations & Number of hospital admissions (6 months prior to enrolment and at 4 and 12 month follow-up \\
& Decisions made per PIP
\end{tabular}

Number of times alternatives were prescribed

Reported primary reason for decision made for example, risks outweigh benefits, patient preference, hospital/consultant initiated

the nature of the intervention, it is not possible to blind GPs or participants to the intervention.

\section{Intervention}

The intervention consists of academic detailing, medicines review with web-based pharmaceutical treatment algorithms and tailored patient information leaflets. The academic detailing will involve a research pharmacist visiting intervention GPs in their own practices. The visit will include a short educational presentation about PIP as a concept, the criteria used to measure it and a summary of studies conducted in Irish primary care on the topic, as knowledge of PIP may be a barrier to appropriate prescribing in older patients [34]. Subsequent to this, practices will be asked to complete ten medicines reviews within a 6 to 8 week period, with a reminder issued if they are not completed within that period. An extended period may be negotiated, should a practice require it. During the medicines review, the GPs will use web-based treatment algorithms specifically designed for this study and accessible using a link and designated password. The algorithm will guide the process from the GP perspective, and does not incorporate patient involvement. It has a page-by-page structure, which will be completed when the GP fills in a review outcome form, detailing decisions made by the GP and patient together, including the reasons for maintaining a PIP, which is a key element of this study. Once the review outcome form has been filled in, the medicines review is complete. The medicines review will take place in the GP practice and will be scheduled at a date and time that is convenient to both the GP and the patient. In group practices where more than one GP is participating, the reviews may be divided between them in a manner that is most suitable to the practice workload. The pilot study indicated that the preparation for the review might be more time consuming for the GP than usual but there was no indication that the consultation itself would be significantly longer. These components are presented in Table 1.

\section{Control group: usual care with simple feedback}

The control-group GPs will continue to provide usual care but will also be provided with simple feedback. Data for patients in the control group will be reviewed during recruitment and a personalized patient list for the ten recruited patients will be given to the GP. The list will summarize the medication class to which the individual patient's potentially inappropriate medication belongs, not the specific PIP and will not provide actionable recommendations for change. Participants will not receive an academic detailing visit, will not be prompted to carry out a medicines review with the individual patients, and will not have access to the pharmaceutical treatment algorithms with alternative therapy options. GPs will continue to provide usual care. In terms of repeat prescribing for public general medical services (GMS) patients, this means that a GP can give a prescription on a monthly basis or for a maximum of three months. At present, the Health Service Executive Primary Care Reimbursement Services (HSE-PCRS) has an on-line GP Application Suite where GPs can review administrative information on their GMS patient panel. They can also access prescribing analysis reports, which 
contain detailed financial and cost information related to their prescribing. Specific prescribing alerts and recommendations for older patients are not provided.

\section{Contemporaneous national control HSE-PCRS dataset}

The control arm will receive simple feedback about their patients based on baseline data collection. Feedback has been found to promote slight improvements in professional practice but is most effective when it is provided intensively $[35,36]$. By participating in this research, the control group might also alter their behaviour, resulting in changes in prescribing patterns (that is, the possible Hawthorne effect). To address this, we will also analyze national prescription patterns for all GMS doctors via the HSE-PCRS prescription database after the trial. This is a national prescribing database based on GP and pharmacy claims in a number of community schemes, including the GMS scheme. Data from the PCRS GMS database can be used to compare practices participating in the study with nonparticipating practices, acting as a contemporary national control. Some 330,000 people aged 70 and over were eligible for the GMS scheme in 2009 [37]. Comparisons with previous PIP patterns nationally [11] will determine whether there have been changes over time at the population level.

\section{Outcome measures}

The study focuses on a select number of PIP criteria identified in the literature that have been determined to be of clinical relevance by academic GPs and pharmacists, and are prevalent in Irish primary care (see Table 2).

The primary outcomes to be determined are the proportion of participant patients with PIP (as a composite measure, that is, any number of PIP criteria as listed in Table 2, to address the issue of multiple PIP in individual patients) and the mean number of potentially inappropriate prescriptions per patient.

Secondary outcomes (summarized in Table 3) will include individual measures of the composite measure, that is, drug-specific outcomes, process evaluations, process-of-care measures and patient-reported outcomes (Health status (EQ-5D) [38], Patients' Beliefs about Medicine Questionnaire (BMQ) [39], Well-being Questionnaire (WBQ-12) [40]).

\section{Data collection}

Prescription data, process-of-care measures and patientreported outcomes will be collected at baseline and on intervention completion, that is, the point at which all ten reviews have been completed (this must be within a 6 to 8 week period). Follow-up data will also be collected 12 months after the intervention completion. Data will be collected from the following sources:

\section{GP medical chart}

Patient records will be used to collect the drug-specific outcome data for all participants at baseline and follow-up. Process-of-care data, such as health-service utilization (for example, the number of GP visits) will also be collected for intervention and control patients.

\section{Questionnaire}

Patient-reported outcomes for intervention and control will come from questionnaire data, which will be collected at baseline and follow-up, using a postal questionnaire and telephone follow-up for nonresponders. The questionnaire will be used to collect personal and demographic data, economic data, and health-service utilization data along with data from the EQ-5D, BMQ and WBQ-12.

\section{Evaluation data}

Process-measure data for the intervention group will be collected by outcome forms completed by GPs at the end of each of the ten medicines reviews they conduct for the ten recruited patients. In single-handed practices, the same GP will conduct the reviews and complete the outcome forms. In group practices, where more than one GP is participating in the study, the reviews may either be conducted by an individual GP nominated by the practice or be shared between the GPs, with GPs completing outcome forms for the patients they reviewed. Semistructured qualitative interviews will also be conducted with both GPs and patients after the intervention, to evaluate the intervention (see below for more detail). GPs in the control arm will also be interviewed, to ascertain any potential impact of the feedback they receive, based on the baseline data.

\section{Plan of analysis}

The minimization process will ensure balance between treatment groups in terms of certain prognostic factors. Descriptive statistics will be used to evaluate differences in other baseline characteristics between participating physicians and patients in the two arms of the trial. The primary analysis will be carried out using multilevel modeling (such as mixed linear effects modeling or generalized estimating equations [41]) to control for the effects of clustering and baseline differences. All analysis will be conducted under the intention-to-treat principle.

Subgroup analyses will be performed for the primary outcome to assess whether the intervention varies by practice size or GP characteristics, such as sex or number of PIP drugs. As this is a pragmatic trial, a secondary, per-protocol analysis will also be conducted. This form of analysis includes only those participants who completed the treatment protocol originally allocated, providing results on the efficacy of the trial [42]. 


\section{Sample size}

As all the patients in this study will be selected on the basis of already having one or more potentially inappropriate prescriptions, the sample size calculations are based on a $100 \%$ prevalence rate. Separate sample size calculations were performed for the two primary outcomes:

\section{Proportion of participant patients with PIP}

The calculation is based on demonstrating a clinically relevant $10 \%$ absolute reduction (from $100 \%$ to $90 \%$ ) in the proportion of PIP with $80 \%$ power and a statistical significance of $5 \%$ (one-sided), between the randomized groups. With a cluster design, the assumption that individual outcomes are independent of each other does not hold, as participants in the same cluster may respond in the same way. The sample size, therefore, needs to be adjusted to reflect this by use of the intraclass correlation coefficient (ICC) [43]. We used an ICC of 0.025, based on an ongoing unpublished observational study of an elderly cohort in the HRB Centre for Primary Care Research. With a maximum of ten patients per cluster and factoring in a loss to follow-up of $10 \%$, a total of 22 GP practices and 212 patients will be required.

\section{Mean number of PIP per patient}

An ongoing unpublished observational study of an elderly cohort in the HRB Centre for Primary Care Research estimates a mean number of 1.45 inappropriate prescriptions per patient. To demonstrate a $30 \%$ relative reduction in the mean number of PIP (equivalent to a mean of 1.02 ), with $80 \%$ power and a statistical significance of $5 \%$ (two-sided), between the randomized groups, with a maximum of ten patients per cluster and factoring in a loss to follow-up of $10 \%$, a total of 14 GP practices and 132 patients will be required.

These calculations indicate that we would need at least 22 practices and 212 patients to detect a difference between the intervention and control arms for both of our primary outcome measures. On the basis of these calculations, we aim to recruit at least 22 practices, with 10 patients per practice, giving a total of 220 patients. With this sample size, we would have at least $80 \%$ power to demonstrate a $10 \%$ absolute reduction in the proportion of PIP and a $30 \%$ relative reduction in the mean number of PIPs. Based on existing evidence that suggests that simple, less intensive feedback does not alter prescribing behaviour $[35,36]$ we have not anticipated an improvement in the control arm. However, we will monitor for this in the parallel process evaluation.

\section{Data management and protection}

A trial steering committee will be established. The aim of the trial is to identify older patients with existing PIP.
These patients will be known to the research team by study ID number only. One member of the research team (BC) will have access to patient contact details for follow-up data collection purposes. The GP remains responsible for all treatment decisions made. Informed consent will be sought from all study participants. All data collected will be stored on a secure, passwordprotected server. All interviews will be audio recorded and transcribed, the digital recording overwritten and the transcripts pseudo-anonymized and stored on a secure, password-protected server.

The academic detailing will demonstrate the process of the medicines review with the intervention practices but the research team will not monitor how the GP implements the study protocol after this, other than to remind the practices to complete the process within the allotted period. This study is pragmatic in nature, measuring the intervention's effectiveness in real clinical practice.

\section{Ethical approval}

Ethical approval was granted by the Research Ethics Committee of the Irish College of General Practitioners (ICGP). At the request of the Ethics Committee, some changes were made to the study protocol. The patient information letter and questionnaire were rewritten to be clearer and simpler for an older audience. Initially, it was proposed that a member of the research team (BC) would become a research agent of the practice, in order to minimize the effort required by the practice staff to recruit patients [44]. However, the ethics committee requested that the practices should be responsible for the patient consent process. The committee also requested that any prescribing pattern of concern identified by the research team should be referred to an external academic GP with no involvement in the trial to assess the case and to determine the necessary next steps; this arrangement has been put in place.

\section{Qualitative evaluation}

A qualitative process evaluation will be conducted to explore participant attitudes towards the intervention and the experience of the intervention delivery. Specifically, semistructured interviews will be conducted with a sample of participants, both GPs and patients, from the intervention arm. The interviews will be structured using a topic guide, which will be developed with the stages of the interview in mind (introducing the research, beginning the interview, and so on) [45]. These interviews will address such research questions as:

1. What are the views of the participants about the acceptability, effectiveness and sustainability of the intervention? 
2. What barriers, if any, were experienced by GPs in relation to implementing the alternative recommended treatments?

3. How did patients respond or react to the idea of altering their medication regimes?

4. Was the medicines review viewed as a useful exercise for the patient or the GP, or both?

5. In what ways might the intervention be modified or adapted to maximize its effectiveness in routine care?

A random sample of participants in the intervention arm will be invited to participate. The number of interviews required to reach data saturation (where no new themes emerge) will be considered, alongside feasibility issues (resources and timing), but a sample of 10 to 15 patients and 10 GPs is proposed [46]. For individual patients, the interviews will take place within one month of the medicines review. For GPs, the interviews will be conducted within one month of them completing their assigned reviews, that is, after all ten reviews for singlehanded practices and after completion of assigned reviews in group practices. The interviews will be conducted either in person (in a setting of the participant's preference) or via telephone. Telephone interviewing is generally used in qualitative research where time or cost is an issue and there is evidence that there is little difference in the answers obtained this way [47]. In this case, scheduling faceto-face interview time with GPs and older patients may be difficult, so telephone interviewing is an option. In addition, as part of the process evaluation, we will conduct brief telephone interviews with the control-group GPs to ascertain any potential impact of the feedback they receive based on the baseline data. Control-group participants will not be required to document any of the decisions made with regard to the control data, so this approach may be more prone to recall bias. However, we consider it a suitable option, given the limited time available in busy GP practices. All interviews will be audio recorded (on loudspeaker for telephone interviews) and transcribed. The data will be collated and a thematic analysis will be conducted. There are four main steps to conducting a thematic analysis:

1. Collect the data.

2. Identify patterns and themes (repetition).

3. Collate related patterns into subthemes.

4. Interpret themes in light of a literature review [48].

NVivo 9 will be used to assist with organizing the data for analysis.

\section{Economic evaluation}

A health economic analysis will be conducted following the RCT to explore the direct costs of the intervention and link these to its potential effectiveness. We will compare the direct costs of delivering the OPTI-SCRIPT intervention as an alternative to usual care. Economic analysis is particularly important in relation to quality of prescribing, owing to the considerable costs invested by healthcare systems in medicines and their prescribing. There are potential cost savings from reducing doses and quantities of inappropriately prescribed medications, and from reducing potential adverse events associated with suboptimal prescribing. There is also potential for cost increases if the appropriate alternative medicine recommended is more expensive. Therefore, it will be important to determine the cost/benefit ratios for any changes made in medicines prescribed as a result of the intervention.

Cost-effectiveness analysis will be undertaken, in which effectiveness will be measured in terms of the reduction in the proportion of potentially inappropriate prescriptions. Direct-cost data will be calculated for all the health resources consumed. All contacts with the health service will be recorded and valued, including GP visits, hospital attendances, hospital admissions and drug prescriptions.

\section{Discussion}

Prescribing for older patients is a complex and challenging task. The literature to date demonstrates that high levels of PIP exist among older people in Ireland [49]. This creates an increased clinical and economic burden with an impact on other patient outcomes, such as increased hospitalizations and mortality. As the proportion of older patients in the population increases and the necessity for pharmaceutical therapy intensifies, it is crucial to find ways to ensure the safety and quality of prescribing in primary care. Currently, no one interventional strategy has proven to be the most effective in addressing PIP. This study is seeking to determine the effectiveness of a complex, multifaceted intervention in reducing the level of PIP in primary care.

The use of the MRC guidelines for the design and evaluation of complex interventions is a strength of this study. The study is innovative in that focuses on a number of PIP criteria that have been determined to be of clinical relevance and high prevalence by academic GPs and pharmacists, rather than applying all criteria from a specific list. Clinically relevant alternatives have also been provided, ensuring that where PIP has been highlighted, actionable recommendations have been made available to the prescriber.

There are some practical limitations to the OPTISCRIPT intervention. The identification of PIP in patients could be carried out by a pharmacist who could apply the criteria to patient records but this would require a formalization of the role of the pharmacist 
within the GP team to enable access to patient records, affecting both cost and service delivery. There are also implications for data protection when a person external to a practice, such as a pharmacist, requires access to patient records. This process could, ideally, be automated, and incorporated into the workflow of the various practice-management software systems used in primary care, along with the treatment algorithms. However, as with all computerized prompts, this would have to be carefully designed to avoid the danger of 'alert fatigue, which could become an issue. In addition, a medicines review process is not standard practice in Irish primary care as it is in other countries, such as the UK, where the National Service Framework for older people recommends that all people over the age of 75 should have their medicines reviewed at least once a year [50]. Were such a process to be introduced in Ireland, an agreement would have to be made about reimbursement mechanisms. The majority of people aged 70 and over in Ireland are entitled to free, state-funded GP care and medications (public patients). A small minority of this age group are private patients (approximately $5 \%$ ), and therefore pay for their own medical care. If a medicines review process were to be introduced into standard care, an agreement would have to be reached as to whether it would be entirely state-funded or whether private patients would have to incur the costs of such a service personally. Should the OPTI-SCRIPT intervention be found to be effective, these issues would need to be taken into consideration prior to its implementation into routine care. In summary, with a growing population of older people, this study will provide evidence concerning the suitability of implementing such an intervention in the Irish Primary Care Sector for older populations.

\section{Trial status}

At the time of submission of this article, 22 GP practices had been recruited. Patient identification and recruitment was just commencing.

\footnotetext{
Abbreviations

ACEl: Angiotensin-converting-enzyme inhibitor; BMQ: Beliefs About Medicine Questionnaire; CME: Continuing medical education; CONSORT: Consolidated Standards of Reporting Trials; COPD: Chronic obstructive pulmonary disease; Gl: Gastro-intestinal; GMS: General medical services; GORD: Gastrooesophageal reflux disease; GP: General practitioner; HRB: Health Research Board; HSE-PCRS: Health Service Executive Primary Care Reimbursement Services; ICC: Intraclass correlation coefficient; ICGP: Irish College of General Practitioners; MRC: Medical Research Council; N/A: Not available; NSAID: Nonsteroidal anti-inflammatory drug; PIP: Potentially inappropriate prescribing; PPI: proton pump inhibitor; p.r.n.: pro re nata, as needed; QUB: Queen's University Belfast; RCSI: Royal College of Surgeons in Ireland; RCT: Randomized controlled trial; SSRI: Selective serotonin reuptake inhibitor; STOPP: Screening Tool of Older People's Prescriptions; TCA: Tricyclic antidepressant; WBQ-12: Well-being Questionnaire.
}

\section{Competing interests}

The authors declare that they have no competing interests.

\section{Authors' contributions}

All authors conceived the development of the intervention and the study design. BC, SS, TF, CH, MB prepared the protocol and contributed to drafting the paper. NM provided statistical advice. RM led the IT design of the study. TF is the principal investigator. The other members of the OPTI-SCRIPT study team are Fiona Boland, Janine Glover and Mary-Claire Kennedy. All authors reviewed and approved the final version of this manuscript.

\section{Acknowledgements}

This study was funded by the HRB PhD Scholars Programme in Health Services Research and the HRB Centre for Primary Care Research, Dublin, Ireland. We wish in particular to acknowledge the contribution made at all stages of this research by Nicola Motterlini who died prior to the submission of this manuscript.

\section{Author details}

${ }^{1}$ Health Research Board (HRB) Centre for Primary Care Research, Royal College of Surgeons in Ireland (RCSI), Beaux Lane House, Lower Mercer Street, Dublin, Ireland. 'SChool of Pharmacy, Queen's University Belfast (QUB), University Road, Belfast Northern BT7 1NN, Ireland. ${ }^{3}$ Department of Geriatric and Stroke Medicine, Royal College of Surgeons in Ireland (RCSI), Beaumont Hospital, Beaumont Road, Dublin 9, Ireland.

Received: 8 August 2012 Accepted: 27 February 2013

Published: 13 March 2013

\section{References}

1. Layte R: Projecting the Impact of Demographic Change on the Demand for and Delivery of Health Care in Ireland. Dublin: The Economic and Social Research Institute; 2009.

2. Barry PJ, Gallagher P, Ryan C: Inappropriate prescribing in geriatric patients. Curr Psychiatry Rep 2008, 10:37-43.

3. Mangoni A, Jackson S: Age-related changes in pharmacokinetics and pharmacodynamics: basic principles and practical applications. $\mathrm{Br} J \mathrm{Clin}$ Pharmacol 2003, 57:6-14.

4. Milton JC, Hill-Smith I, Jackson SH: Prescribing for older people. BMJ 2008, 336:606-609.

5. Page RL, Linnebur SA, Bryant $L L$, Ruscin JM: Inappropriate prescribing in the hospitalized elderly patient: defining the problem, evaluation tools, and possible solutions. Clin Interv Aging 2010, 5:75-87.

6. Gallagher P, Barry P, O'Mahony D: Inappropriate prescribing in the elderly. J Clin Pharm Ther 2007, 32:113-121.

7. Lau DT, Kasper JD, Potter DE, Lyles A, Bennett RG: Hospitalization and death associated with potentially inappropriate medication prescriptions among elderly nursing home residents. Arch Intern Med 2005, 165:68-74.

8. Lin HY, Liao CC, Cheng SH, Wang PC, Hsueh YS: Association of potentially inappropriate medication use with adverse outcomes in ambulatory elderly patients with chronic diseases: experience in a Taiwanese medical setting. Drugs Aging 2008, 25:49-59.

9. Spinewine A, Schmader K, Barber N, Hughes C, Lapane K, Swine C, Hanlon J: Appropriate prescribing in elderly people: how well can it be measured and optimised? Lancet 2007, 370:173-184.

10. Gallagher P, O'Mahony D: STOPP (Screening Tool of Older Persons' Potentially Inappropriate Prescriptions) application to acutely ill elderly patients and comparison with Beers' criteria. Age Ageing 2008, 37:673-679.

11. Cahir C, Fahey T, Teeling M, Teljeur C, Feely J, Bennett K: Potentially inappropriate prescribing and cost outcomes for older people: a national population study. Br J Clin Pharmacol 2010, 69:543-552.

12. Oxman $A D$, Fretheim $A$, Flottorp $S$ : The OFF theory of research utilization. J Clin Epidemiol 2005, 58:113-116.

13. Bhattacharyya O, Reeves S, Garfinkel S, Zwarenstein M: Designing theoretically-informed implementation interventions: fine in theory, but evidence of effectiveness in practice is needed. Implement Sci 2006, 1:5.

14. Majumdar SR, Lipton HL, Soumerai SB: Evaluating and Improving Physician Prescribing in Pharmacoepidemiology. 4th edition. Chichester: Wiley; 2005.

15. Marcum ZA, Handler SM, Wright R, Hanlon JT: Interventions to improve suboptimal prescribing in nursing homes: a narrative review. Am J Geriatr Pharmacother 2010, 8:183-200.

16. O' Brien MA, Rogers $S$, Jamtvedt G, Oxman AD, Odgaard-Jensen J, Kristofferson DT, Forsetlund L, Bainbridge D, Freemantle N, Davis DA, 
Haynes RB, Harvey EL: Educational outreach visits: effects on professional practice and health care outcomes. Cochrane Database Syst Rev 2007, 4:CD000409.

17. Castelino RL, Bajorek BV, Chen TF: Targeting suboptimal prescribing in the elderly: a review of the impact of pharmacy services. Ann Pharmacother 2009, 43:1096-1106.

18. Geurts MME, Talsma J, Brouwers JRBJ, de Gier JJ: Medication review and reconciliation with cooperation between pharmacist and general practitioner and the benefit for the patient: a systematic review. $\mathrm{Br} J \mathrm{Clin}$ Pharmacol 2012, 74:16-33.

19. Kaur S, Mitchell G, Vitetta L, Roberts MS: Interventions that can reduce inappropriate prescribing in the elderly: a systematic review. Drugs Aging 2009, 26:1013-1028.

20. Garcia RM: Five ways you can reduce inappropriate prescribing in the elderly: a systematic review. J Fam Pract 2006, 55:305-312.

21. Blenkinsopp A, Bond C, Raynor DK: Medication reviews. Br J Clin Pharmacol 2012, 74:573-580

22. Ostini R, Hegney D, Jackson C, Williamson M, Mackson JM, Gurman K, Hall W, Tett SE: Systematic review of interventions to improve prescribing. Ann Pharmacother 2009, 43:502-513.

23. Robben S, van Kempen J, Heinen M, Zuidema S, Olde Rikkert M, Schers H, Melis R: Preferences for receiving information among frail older adults and their informal caregivers: a qualitative study. Fam Pract 2012, 29:742-747.

24. Kenny T, Wilson RG, Purves IN, Clark J, Newton LD, Newton DP, Moseley DV: A PIL for every ill? Patient information leaflets (PILs): a review of past, present and future use. Fam Pract 1998, 15:471-479.

25. Majumdar SR, Soumerai SB: Why most interventions to improve physician prescribing do not seem to work. CMAJ 2003, 169:30-31.

26. Grimshaw JM, Shirran L, Thomas R, Mowatt G, Fraser C, Bero L, Grilli R, Harvey E, Oxman A, O'Brien MA: Changing provider behavior: an overview of systematic reviews of interventions. Med Care 2001, 39(8 Suppl 2):|12-1|145.

27. Craig P, Dieppe P, Macintyre S, Michie S, Nazareth I, Petticrew M: Developing and evaluating complex interventions: the new Medical Research Council guidance. BMJ 2008, 337:a1655.

28. Medical Research Council: A Framework for Development and Evaluation of RCTs for Complex Interventions to Improve Health. London; 2000.

29. Lewin S, Glenton C, Oxman AD: Use of qualitative methods alongside randomised controlled trials of complex healthcare interventions: methodological study. BMJ 2009, 339:b3496.

30. Campbell MK, Elbourne DR, Altman DG: CONSORT statement: extension to cluster randomised trials. BMJ 2004, 328:702-708.

31. Eldridge SM, Ashby D, Feder GS, Rudnicka AR, Ukoumunne OC: Lessons for cluster randomized trials in the twenty-first century: a systematic review of trials in primary care. Clin Trials 2004, 1:80-90.

32. Torgerson DJ, Torgerson CJ: Designing Randomised Trials in Health, Education and the Social Sciences. Basingstoke, Hampshire: Palgrave Macmillan; 2008.

33. O' Dowd T, O' Kelly M, O' Kelly F: Structure of General Practice in Ireland 1982-2005. Dublin: Trinity College Dublin; 2006.

34. Ramaswamy R, Maio V, Diamond JJ, Talati AR, Hartmann CW, Arenson C, Roehl B: Potentially inappropriate prescribing in elderly: assessing doctor knowledge, confidence and barriers. J Eval Clin Pract 2011, 17:1153-1159.

35. Jamtvedt G, Young J, Kristofferson DT, O' Brien MA, Oxman AD: Audit and feedback: effects on professional practice and health care outcomes. Cochrane Database Syst Rev 2006, 2:CD000259.

36. Ivers N, Jamtvedt G, Flottorp S, Young J, Odgaard-Jensen J, French S, O'Brien MA, Johansen M, Grimshaw J, Oxman AD: Audit and feedback: effects on professional practice and healthcare outcomes. Cochrane Database Syst Rev 2012, 6:CD000259.

37. Health Service Executive: Primary Care Reimbursement Service Statistical Analysis Of Claims and Payments 2009. Dublin; 2009

38. The EuroQol Group: EuroQol - a new facility for the measurement of health-related quality of life. Health Policy 1990, 16:199-208.

39. Horne R, Weinman J, Hankins M: The Beliefs About Medicines questionnaire: the development and evaluation of a new method for assessing the cognitive representation of medication. Psychol Health 1999, 14:1-24.

40. Pouwer F, van der Ploeg HM, Adèr HJ, Heine RJ, Snoek FJ: The 12-item Well-being Questionnaire. An evaluation of its validity and reliability in Dutch people with diabetes. Diabetes Care 1999, 22:2004-2010.
41. Campbell MK, Mollison J, Steen N, Grimshaw JM, Eccles M: Analysis of cluster randomized trials in primary care: a practical approach. Fam Pract 2000, 17:192-196.

42. Jadad A: Randomised Controlled Trials: A User's Guide. London: BMJ Books; 1998.

43. Campbell MK, Thomson S, Ramsay CR, MacLennan GS, Grimshaw JM: Sample size calculator for cluster randomized trials. Comput Biol Med 2004, 34:113-125.

44. Page M, French S, McKenzie J, O'Connor D, Green S: Recruitment difficulties in a primary care cluster randomised trial: investigating factors contributing to general practitioners' recruitment of patients. BMC Med Res Methodol 2011, 11:35.

45. Ritchie J, Lewis J: Qualitative Research Practice: A Guide for Social Science Students and Researchers. London: Sage; 2010.

46. Morse JM: Determining sample size. Qual Health Res 2000, 10:3-5.

47. Sturges JE, Hanrahan KJ: Comparing telephone and face-to-face qualitative interviewing: a research note. Qual Res 2004, 4:107-118.

48. Aronson J: A pragmatic view of thematic analysis. The Qualitative Report 1994, 2 [http://www.nova.edu/ssss/QR/Backlssues/QR2-1/aronson.html].

49. Ryan C, O'Mahony D, Kennedy J, Weedle P, Byrne S: Potentially inappropriate prescribing in an Irish elderly population in primary care. Br J Clin Pharmacol 2009, 68:936-947.

50. Department of Health: National Service Framework for Older People. London; 2001.

\section{doi:10.1186/1745-6215-14-72}

Cite this article as: Clyne et al:: Effectiveness of medicines review with web-based pharmaceutical treatment algorithms in reducing potentially inappropriate prescribing in older people in primary care: a cluster randomized trial (OPTI-SCRIPT study protocol). Trials 2013 14:72.

\section{Submit your next manuscript to BioMed Central and take full advantage of:}

- Convenient online submission

- Thorough peer review

- No space constraints or color figure charges

- Immediate publication on acceptance

- Inclusion in PubMed, CAS, Scopus and Google Scholar

- Research which is freely available for redistribution

Submit your manuscript at www.biomedcentral.com/submit
Ciomed Central 